\title{
Applications of Tensor Models in Wireless Communications and Mobile Computing
}

\author{
C. Alexandre R. Fernandes $\mathbb{I D}^{1},{ }^{1}$ Jianhe Du $\mathbb{D}^{2},{ }^{2}$ Alex P. da Silva, ${ }^{3}$ and André L. F. de Almeida $\mathbb{D}^{1}$ \\ ${ }^{1}$ Federal University of Ceará (UFC), Fortaleza, Brazil \\ ${ }^{2}$ Communication University of China, Beijing, China \\ ${ }^{3}$ Alternative Energies and Atomic Energy Commission (CEA), Grenoble, France
}

Correspondence should be addressed to C. Alexandre R. Fernandes; alexandrefernandes@ufc.br

Received 28 January 2020; Accepted 28 January 2020; Published 20 February 2020

Copyright (c) 2020 C. Alexandre R. Fernandes et al. This is an open access article distributed under the Creative Commons Attribution License, which permits unrestricted use, distribution, and reproduction in any medium, provided the original work is properly cited.

Tensor decompositions, also named tensor factorizations, are very useful tools for representing and analyzing multidimensional data in a compact way. In some cases, tensor decompositions can be viewed as generalizations of matrix decompositions, such as the singular value decomposition (SVD), to higher-order arrays. In addition to enabling multidimensional data processing, tensor models have properties that become possible to solve undetermined solution problems under conditions more relaxed than conventional matrix approaches [1-3].

Recent developments in multilinear algebra have made possible the application of tensor analysis in several areas. In particular, tensor approaches have gained considerable space in wireless communications and mobile networks [4]. The proposition of new factorizations of tensors, as well the study of uniqueness and identifiability conditions, has a great potential to provide remarkable improvements in these fields.

Indeed, in the literature, one can find a wide range of applications of tensor and multilinear algebra in problems such as Internet traffic [5], missing data problems [6], bigdata analysis [7], neuron networks [8], image processing [9], design of mobile communication systems [10, 11], and development of receivers for wireless systems [12].

Although much research has been done within this subject, many challenges are still to be explored. The proposition of new tensor decompositions, factorization algorithms, and mathematical properties has a great potential to bring significant impacts in several areas. The purpose of this special issue is to publish original efforts presenting recent advances in tensor decompositions with applications in communication systems and mobile computing.

B. Kurt et al. propose the use of tensor techniques for modeling network flows, which is exploited to solve recovery information problems. The novelty of this work consists in the first application of multiway methods for network monitoring, being an interesting alternative for recovering the true flow length distribution from the sampled traffic data. The data is modeled as a three-way array, with each characteristic being estimated using a nonnegative tensor factorization. The proposed tensor factorization scheme is validated with synthetic data and applied to real-world data with two different sampling methods.

P. R. B. Gomes et al. address the problem of joint downlink (DL) and uplink (UL) channel estimation for millimeter wave (mmWave) multiple-input multiple-output (MIMO) systems and develop two tensor-based semiblind receivers by capitalizing on the multilinear structure and sparse nature of the received signal at the BS equipped with a hybrid analog-digital beamforming (HB) architecture. This paper shows the benefits of jointly estimating both the DL and UL channels as a single problem by concentrating most of the processing burden for channel estimation at the BS side, which are attractive features for massive MIMO systems.

L. Liu et al. present a new algorithm to estimate the direction of arrival (DOA) and polarization parameters of signals impinging on an array with electromagnetic (EM) vector-sensors by exploiting the canonical polyadic 
decomposition (CPD) of tensors. In addition to spatial and temporal diversities, further information from the polarization domain is exploited. The authors show that the parameters can be estimated by virtue of the diversities of the spatial and polarization belonging to the factor matrices, rather than using conventional subspace methods.

M. T. de Oliveira et al. address a practical DOA problem by proposing a low-cost antenna array-based drone tracking device for outdoor environments. The proposed solution is divided into hardware and software parts. The hardware part of the proposed device is based on off-the-shelf components such as an omnidirectional antenna array, a 4-channel software defined radio (SDR) platform with carrier frequency ranging from $70 \mathrm{MHz}$ to $6 \mathrm{GHz}$, a FPGA motherboard, and a laptop. The software part includes algorithms for calibration, model order selection (MOS), and DOA estimation, including specific preprocessing steps and a tensor-based estimator to increase the DOA accuracy.

With this special issue, we hope that readers will be interested in applications of tensor decompositions and they will find this issue helpful for their research.

\section{Conflicts of Interest}

The editors have no conflicts of interest to the assigned manuscripts when handling them and making decisions.

\section{Alexandre R. Fernandes Jianhe Du Alex P. da Silva André L. F. de Almeida}

[8] W. Hong, W. Xu, J. Qi, and Y. Weng, "Neural tensor network for multi- label classification," IEEE Access, vol. 7, pp. 96936-96941, 2019.

[9] J. Wu, Z. Lin, and H. Zha, "Essential tensor learning for multiview spectral clustering," IEEE Transactions on Image Processing, vol. 28, no. 12, pp. 5910-5922, 2019.

[10] G. Favier, C. A. R. Fernandes, and A. L. F. de Almeida, "Nested Tucker tensor decomposition with application to MIMO relay systems using tensor space-time coding (TSTC)," Signal Processing, vol. 128, pp. 318-331, 2016.

[11] D. S. Rocha, G. Favier, and C. A. R. Fernandes, “Tensor coding for three-hop MIMO relay systems," in roceedings of the IEEE Symposium on Computers and Communications (ISCC), Natal, Brazil, 2018.

[12] W. d. C. Freitas Jr., G. Favier, and A. L. F. de Almeida, "Sequential closed-form semiblind receiver for space-time coded multihop relaying systems," IEEE Signal Processing Letters, vol. 24, no. 12, pp. 1773-1777, 2017.

\section{References}

[1] A. Cichoki, D. Mandic, L. de Lathawer et al., "Tensor decompositions for signal processing applications: from twoway to multiway component analysis," IEEE Signal Processing Magazine, vol. 32, no. 2, pp. 145-163, 2015.

[2] T. G. Kolda and B. W. Bader, "Tensor decompositions and applications," SIAM Review, vol. 51, no. 3, pp. 455-500, 2009.

[3] P. Comom, "Tensor decompositions-state of the art and applications," in Proceedings of the MA Conference Mathematics in Signal Processing, Warwick, UK, 2000.

[4] A. L. F. de Almeida, G. Favier, J. P. C. L. da Costa, and J. C. M. Mota, "Overview of tensor decompositions with applications to communications," in Signals and Images: Advances and Results in Speech, Estimation, Compression, Recognition, filtering, and Processing, pp. 325-356, CRC Book, Boca Raton, FL, USA, 2016.

[5] K. Xie, C. Peng, X. Wang et al., "Accurate recovery of internet traffic data under variable rate measurements," IEEE/ACM Transactions on Networking, vol. 26, no. 3, pp. 1137-1150, 2018.

[6] K. Xie, L. Wang, X. Wang et al., "Accurate recovery of internet traffic data: a sequential tensor completion approach," IEEE/ ACM Transactions on Networking, vol. 26, no. 2, pp. 793-806, 2018.

[7] A. Cichocki, "Tensor networks for big data analytics and largescale optimization problems," 2014, https://arxiv.org/abs/ 1407.3124. 